\title{
Thermal emission from finite photonic crystals
}

\author{
Christian J. Schuler, ${ }^{1}$ Christian Wolff, ${ }^{1}$ Kurt Busch, ${ }^{1}$ and Marian Florescu ${ }^{2, a)}$ \\ ${ }_{1}^{1}$ Institut für Theoretische Festkörperphysik and DFG-Center for Functional Nanostructures (CFN), \\ Karlsruhe Institute of Technology, 76128 Karlsruhe, Germany \\ ${ }^{2}$ Department of Physics, Princeton University, Princeton, New Jersey 08544, USA
}

(Received 3 November 2009; accepted 22 November 2009; published online 16 December 2009)

\begin{abstract}
We present a microscopic theory of thermal emission from finite-sized photonic crystals and show that the directional spectral emissivity and related quantities can be evaluated via standard bandstructure computations without any approximation. We then identify the physical mechanisms through which interfaces modify the potentially super-Planckian radiation flow inside infinite photonic crystals, such that thermal emission from finite-sized samples is consistent with the fundamental limits set by Planck's law. As an application, we further demonstrate that a judicious choice of a photonic crystal's surface termination facilitates considerable control over both the spectral and angular thermal emission properties. (c) 2009 American Institute of Physics.
\end{abstract}

[doi:10.1063/1.3275578]

Photonic crystals (PhCs) are widely recognized for offering the potential to controllably alter light-matter interaction and light propagation on a fundamental level. ${ }^{1-3}$ Early theoretical works have already appreciated the profound modifications of the fluorescence properties of individual emitters embedded in $\mathrm{PhCs}^{4}$ but despite significant progress, experimental investigations of such systems remain rather challenging. Experimentally more accessible is thermally driven fluorescence, i.e., thermal emission from emitters inside PhCs. ${ }^{5-7}$ Besides the scientific relevance of the investigation of thermal emission from PhCs, numerous technological applications, such as thermophotovoltaic energy conversion devices and light sources, will benefit from a detailed understanding of the underlying physics. ${ }^{7-9}$

In the present letter, we develop a microscopic theory for thermal emission in finite PhCs that are in thermal equilibrium. Our formalism relies solely on a natural modal expansion of the electromagnetic field that can be related to standard photonic bandstructure computations without invoking any approximation. Such a general framework enables several significant advances. First, while the previous results for infinite $\mathrm{PhCs}$ show that the thermal radiation intensity may exceed the free-space blackbody (BB) limit, ${ }^{10,11}$ it is, on thermodynamic grounds ${ }^{8}$ (verified by direct simulation ${ }^{12,13}$ ), clear that the introduction of an interface must limit the maximally allowed thermal emission from finite-sized $\mathrm{PhCs}$ to the BB limit set by Planck's law. Second, regarding more practical aspects, our formalism allows to compute the spectral and angular characteristics of thermal emission from $\mathrm{PhCs}$ "bottom-up" (i.e., without invoking Kirchhoff's law or any variation thereof) and can be used to design future applications based on the ability to engineer electromagnetic mode properties such as group velocity and reflection at interfaces.

We consider a half-space problem where a semi-infinite, lossless dielectric $\mathrm{PhC}$ is separated from free space by a planar interface. This system is in thermal equilibrium at temperature $T$. The periodicity of the infinite $\mathrm{PhC}$ gives rise to propagating Bloch modes with frequency $\omega_{n, \mathbf{k}}$, where $n$ and

${ }^{a)}$ Electronic mail: florescu@princeton.edu. $\mathbf{k}$, respectively, label the band index and (real-valued) wave vector associated with a given mode. The wave vector is confined to the first Brillouin zone. In thermal equilibrium, emitters that are embedded in the $\mathrm{PhC}$ populate the available propagating Bloch modes and it is readily seen ${ }^{10,11}$ that their occupation number obeys Bose-Einstein statistics $\bar{n}(\omega, T)$ $=\left(e^{\hbar \omega / k_{B} T}-1\right)^{-1}$. The excited Bloch modes can couple to free space and this will lead to the emission of radiation into the far field of the propagating Bragg orders. We compute the transmittance coefficients via a generalization of an on-shell (fixed frequency $\omega$ ) bandstructure method that allows for real- and complex-valued wave vectors, i.e., propagating and evanescent Bloch modes. ${ }^{14}$ Within our approach, the planewave expansion of a Bloch mode $\{n, \mathbf{k}\}$ into the reciprocal lattice vectors of the infinite PhC's Bravais lattice, is rearranged as an expansion into plane waves $\mathbf{G}_{\|}$that are obtained by stripping each reciprocal lattice vector $\mathbf{G}$ of its component normal to the surface. On the air side, a corresponding expansion of the electromagnetic field into the same set of plane waves $\mathbf{G}_{\|}$is employed. Upon splitting the Bloch mode's wave vector $\mathbf{k}_{n}$ into parallel and perpendicular contributions, $\mathbf{k}_{\|, n}$ and $\mathbf{k}_{\perp, n}$, the continuity of the tangential electric and magnetic fields across the interface can be enforced by comparing the terms that belong to different plane waves $\mathbf{G}_{\|}$.

In general, an excited Bloch mode $\{n, \mathbf{k}\}$ will transmit energy into several Bragg orders so that the corresponding time-averaged Poynting vector on the air side is

$$
\mathbf{S}_{n, \mathbf{k}}^{\mathrm{air}}=\sum_{G_{\|}} \mathbf{S}_{n, \mathbf{k}}^{\mathrm{air}, \mathbf{k}_{G_{\|}}^{\prime}}
$$

Here, each summand contains the square of the transmittance coefficient of the excited Bloch mode $n, \mathbf{k}$ into the corresponding propagating Bragg order that is characterized by its surface-parallel wave vector $\mathbf{k}_{\|}^{\prime}=\mathbf{k}_{\|, n}+\mathbf{G}_{\|}$. Since the total wave number of such a propagating Bragg order is $\omega / c$, we can also determine the corresponding real-space direction of energy flow in terms of the angle $\phi$ relative to the surface normal as $\left|\mathbf{k}_{\|}^{\prime}\right|=(\omega / c) \sin \phi$.

In order to determine the energy density current on the air side that is generated by the excited Bloch mode $\{n, \mathbf{k}\}$, 

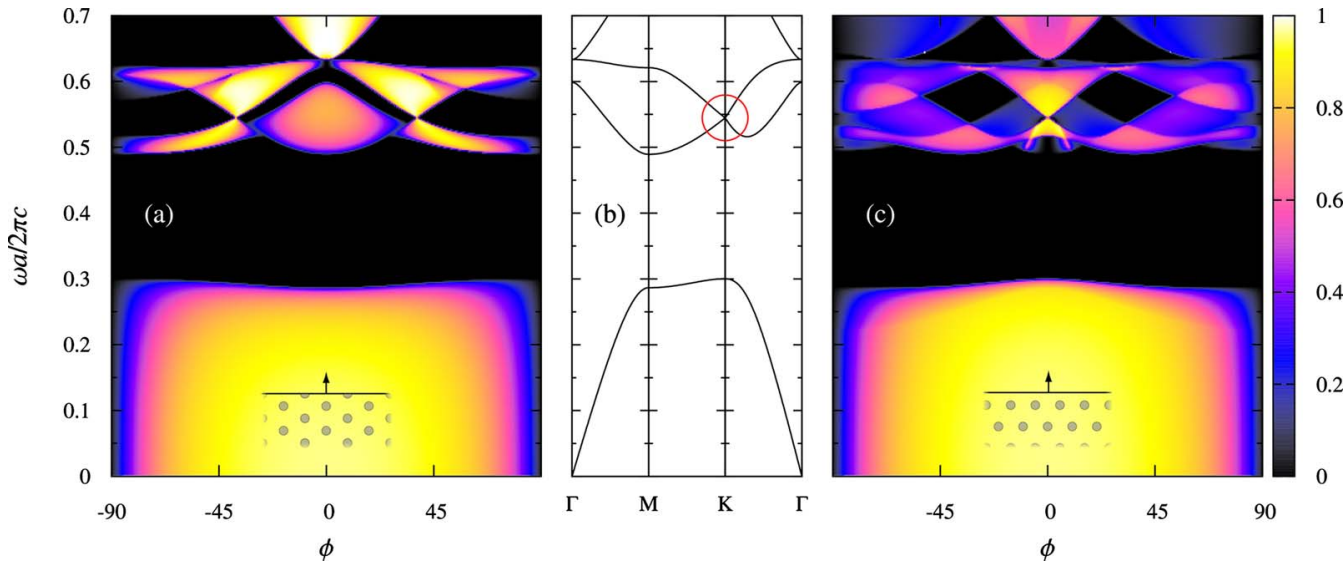

FIG. 1. (Color online) DSE from a 2D hexagonal lattice of dielectric rods in air, E-polarized radiation. The plane of the interface is perpendicular to (a) the $\Gamma-\mathrm{M}$ or $(\mathrm{c})$ the $\Gamma$-K direction. The corresponding photonic bandstructure is shown in (b).

we have to weight the Poynting vector, Eq. (1), with the (directional) density of states $\rho\left(\omega, \mathbf{k}_{\|, n}\right)=\left[(2 \pi)^{2} v_{\perp, n, \mathbf{k}}\right]^{-1}$ along the isofrequency surface, i.e., at frequency $\omega$ along the parallel wave vector $\mathbf{k}_{\|, n}$. Here $v_{\perp, n, \mathbf{k}}$ denotes the group velocity component that is perpendicular to the surface and can be obtained via standard bandstructure computations. ${ }^{15}$ Consequently, the energy current density that the Bloch modes around $\{n, \mathbf{k}\}$ emit into the propagating Bragg orders is

$$
\mathbf{J}_{n, \mathbf{k}}=\sum_{G_{\|}} \frac{1}{(2 \pi)^{2}} \frac{1}{v_{n, \mathbf{k}, \perp}} \mathbf{S}_{n, \mathbf{k}}^{\mathrm{air}, \mathbf{k}_{G_{\|}}^{\prime}} d k_{\|} .
$$

For a plane wave in air, we have that the infinitesimal angle $d \phi$ around $\phi$ is $d \phi=d k_{\|}^{\prime} / k_{\perp, G_{\|}}^{\prime}\left(\right.$ where $k_{\perp, G_{\|}}^{\prime}=\left(\omega^{2} / c^{2}\right.$ $\left.-\left|\mathbf{k}_{\|}^{\prime}\right|^{2}\right)^{1 / 2}$ ) so that we may express Eq. (2) in terms of the real space direction described by the angle $\phi$.

Finally, we obtain the directional spectral intensity $i_{\mathrm{PhC}}(\omega, \phi, T)$ by collecting the contributions from all modes associated with a certain emission angle $\phi$ and weighting them by the occupation $\bar{n}(\omega, T)$ of the modes

$$
i_{\mathrm{PhC}}(\omega, \phi, T)=\sum_{\{\mathbf{k}, n\}, G_{\|}} \frac{\bar{n}(\omega, T)}{(2 \pi)^{2}} \frac{k_{\perp, G_{\|}}^{\prime}}{v_{\perp, n, \mathbf{k}}}\left|\mathbf{S}_{n, \mathbf{k}}^{\mathrm{air}, \phi}\right| .
$$

More precisely, Eq. (3) represents the energy flux from the $\mathrm{PhC}$ into the infinitesimal angle around $\phi$ on the air side within the frequency interval $[d \omega, \omega+d \omega]$ and at temperature $T$. The summation in Eq. (3) is restricted such that $\mathbf{k}_{\|}^{\prime}=\mathbf{k}_{\|, n}$ $+\mathbf{G}_{\|}$is fulfilled and $\left|\mathbf{k}_{\|}^{\prime}\right|=(\omega / c) \sin \phi$ corresponds to the emission angle $\phi$. If we normalize the directional spectral intensity [Eq. (3)] to that of a $\mathrm{BB}$, we obtain the directional spectral emissivity (DSE)

$$
\varepsilon(\omega, \phi)=\frac{i_{\mathrm{PhC}}(\omega, \phi, T)}{\left(\hbar \omega^{2} / 4 \pi^{2} c\right) /\left(e^{\hbar \omega / k_{B} T}-1\right)} .
$$

The DSE is a measure of the thermal radiation flux of a given structure relative to the one emitted by a BB. If we assume that the PhC's bandstructure remains unaltered by temperature, the DSE is temperature independent.

In the following, a two-dimensional (2D) model $\mathrm{PhC}$ will help to illustrate several features of thermal emission that are generic to PhCs. We consider a semi-infinite hexagonal lattice of silicon rods (dielectric constant $\epsilon=11.56$ and radius $r / a=0.176)$ in air where emitters populate the E-polarized modes. Besides a photonic band gap (PBG) this structure exhibits in its bandstructure a Dirac-point that leads to unusual transport properties in the presence of disorder. ${ }^{16}$ We display the corresponding bandstructure in Fig. 1(b) along with the DSE for two distinct surface terminations, Figs. 1(a) and 1(c).

Consistent with thermodynamics, the DSE is bounded from above by unity for all frequencies and angles. In the long-wavelength limit, the $\mathrm{PhC}$ behaves as an effective (homogeneous) medium with a refractive index $n_{\text {eff }}$ so that the angular behavior is dominated by refraction and total internal reflection. At higher frequencies, we observe a strong angular dependence; for frequencies just below the lower edge of the $\mathrm{PBG}$, the $\Gamma-\mathrm{K}$ termination leads to very strong emission for very large angles up to $90^{\circ}$. This behavior is the remnant of the super-Planckian emission inside the infinite $\mathrm{PhC}$ for frequencies near the first stop band. ${ }^{10,11}$ For frequencies just above the lower edge of the PBG, we find that the $\Gamma-\mathrm{M}$ termination leads to a preferred emission into large angles, while the $\Gamma-\mathrm{K}$ termination induces a pronounced forward emission. As a matter of fact, these effects can be directly linked to the radiation focusing effects of the infinite $\mathrm{PhC}$ near the PBG of the model system. ${ }^{10,11}$ Moreover, we observe that for frequencies near this Dirac point $(a / \lambda$ $\approx 0.545$ ) the thermal emission for a $\Gamma$-M terminated $\mathrm{PhC}$ is effectively channeled into the angular range around $\pm 37.5^{\circ}$ whereas for a $\Gamma-\mathrm{K}$ terminated $\mathrm{PhC}$ this channeling occurs into the forward direction over a large frequency range (emission limited to a $\pm 23^{\circ}$ window over a frequency range of about $15 \%$ around the frequency of the Dirac point). These results demonstrate that a judicious choice of the surface termination facilitates far-reaching control of the thermal emission characteristics.

Next, we connect our results of our "bottom-up" DSE calculations with those of a "top-down" approach where the directional spectral absorptance is calculated in an independent way. ${ }^{13}$ Instead of exciting modes in the $\mathrm{PhC}$ and tracking their behavior at the $\mathrm{PhC}$-air interface, we consider the reverse situation and analyze (with our generalization of the method of Ref. 14) the scattering of plane waves at the PhC structure surface. Since the $\mathrm{PhC}$ is semi-infinite, we have to treat all the radiation that is transmitted into the $\mathrm{PhC}$ as being absorbed by the $\mathrm{PhC}$, so that the absorptance $\alpha(\omega, \phi)=1$ $-r(\omega, \phi)$ for a plane wave (with frequency $\omega$ and angle $\phi$ to the surface normal) is given by in terms of the total reflec- 


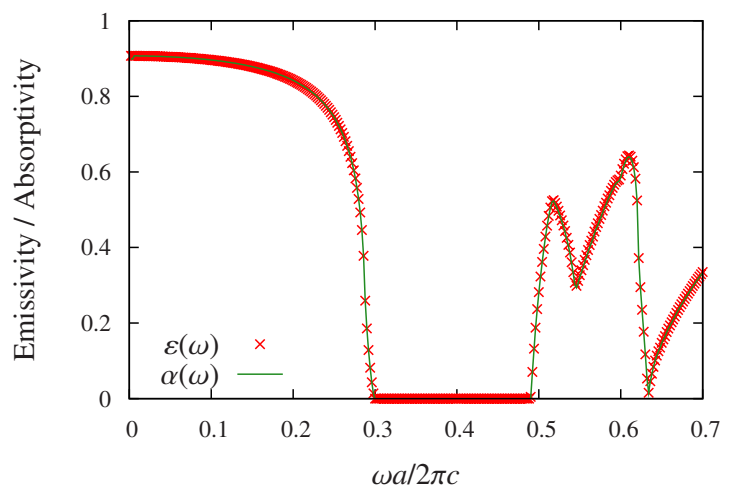

FIG. 2. (Color online) Hemispherical spectral emissivity (solid line) and absorptivity (symbols) from a 2D hexagonal lattice of dielectric rods in air, E-polarized radiation.

tance $r(\omega, \phi)$. Next, we evaluate the hemispherical spectral emissivity $\varepsilon(\omega)$ [absorptivity $\alpha(\omega)$ ] by performing an angular integration over the corresponding directional quantities. In Fig. 2, we demonstrate that hemispherical spectral emissivity equals absorptivity. Actually, this equality also holds for the directional spectral emissivities and absorptivities for arbitrary angles (not shown). In other words, Kirchhoff's law is a consequence of our microscopic theory and not part of its conceptual basis.

In summary, we have developed a microscopic theoretical framework for analyzing the thermal emission from finite $\mathrm{PhC}$ samples in thermal equilibrium. Without any further assumptions, this framework obeys the fundamental laws of thermodynamics. Therefore, this theory represents an ideal starting point for an analysis of the nonequilibrium properties of such systems. With this "bottom-up" framework, we have further demonstrated that the spectral as well as angular thermal emission characteristics from $\mathrm{PhCs}$ may be controlled over a wide range via a combination of focusing ef- fects associated with the infinite $\mathrm{PhC}$ and the PhC's surface termination. In turn, this suggests numerous applications for high-efficiency and highly directional light sources as well as for optimized thermophotovoltaic setups where the thermal emission properties have to be matched to the properties of available low-cost photovoltaic cells.

C.W. and K.B. acknowledge the support by the Deutsche Forschungsgemeinschaft and the State of BadenWürttemberg through the DFG-Center for Functional Nanostructures within project A1.1. The Ph.D. education of C.W. is embedded in Karlsruhe School of Optics \& Photonics (KSOP). C.J.S. acknowledges support by the DAAD.

${ }^{1}$ S. John, Phys. Rev. Lett. 58, 2486 (1987).

${ }^{2}$ E. Yablonovitch, Phys. Rev. Lett. 58, 2059 (1987).

${ }^{3}$ K. Busch, G. von Freymann, S. Linden, S. F. Mingaeleev, L. Tkeshelashvili, and M. Wegener, Phys. Rep. 444, 101 (2007).

${ }^{4}$ S. John and T. Quang, Phys. Rev. A 50, 1764 (1994).

${ }^{5}$ J. G. Fleming, S.-Y. Lin, I. El-Kady, R. Biswas, and K.-M. Ho, Nature (London) 417, 52 (2002).

${ }^{6}$ S.-Y. Lin, J. G. Fleming, and E. El-Kady, Opt. Lett. 28, 1909 (2003).

${ }^{7}$ S.-Y. Lin, J. Moreno, and J. G. Fleming, Appl. Phys. Lett. 83, 380 (2003).

${ }^{8}$ T. Trupke, P. Würfel, and M. A. Green, Appl. Phys. Lett. 84, 1997 (2004).

${ }^{9}$ M. Florescu, H. Lee, I. Puscasu, M. Pralle, L. Florescu, D. Z. Ting, and J. P. Dowling, Sol. Energy Mater. Sol. Cells 91, 1599 (2007).

${ }^{10}$ M. Florescu, K. Busch, and J. P. Dowling, Phys. Rev. B 75, 201101(R) (2007).

${ }^{11}$ M. Florescu and K. Busch, J. Opt. A, Pure Appl. Opt. 11, 114005 (2009).

${ }^{12}$ C. Luo, A. Narayanaswamy, G. Chen, and J. D. Joannopoulos, Phys. Rev. Lett. 93, 213905 (2004).

${ }^{13}$ D. L. Chan, M. Soljačić, and J. D. Joannopoulos, Phys. Rev. E 74, 036615 (2006).

${ }^{14}$ C. S. Feng, L. M. Mei, L. Z. Cai, X. L. Yang, S. S. Wei, and P. Li, J. Phys. D 39, 4316 (2006).

${ }^{15}$ D. Hermann, M. Frank, K. Busch, and P. Wölfle, Opt. Express 8, 167 (2001)

${ }^{16}$ R. A. Sepkhanov and C. W. J. Beenakker, Opt. Commun. 281, 5267 (2008). 